\title{
LÉXICO Y CONTEXTO SITUACIONAL EN EL IDEARIO POÉTICO DE GARY SNYDER
}

M" Carmen Fernández Leal

El propósito que nos impulsa a escribir el presente artículo tiene su fundamento en la búsqueda de una posible identidad entre la expresión formal de una idea y los medios que para ello se utilizan: su representación por medio de la frase y sus componentes, siendo analizados desde una perspectiva semántica, a la que va aparejada la contrapartida sintáctica. Hacemos resaltar los conceptos estructurales de espacio, tiempo y movimiento, como pilares sobre los que se asienta la expresión de una idea, así como el de función, que pone de manifiesto el tipo de relación que se establece entre los términos léxicos que se utilizan para la formulación de un juicio en el marco de una situación determinada.

El 'corpus' que hemos seleccionado para ser analizado proviene de la obra literaria del poeta americano Gary Snyder, y abarca una parte de los poemas agrupados bajo el epígrafe de 'Manzanita', que fueron publicados por primera vez en $1972 \mathrm{e}$ incluidos, posteriormente, en el libro de poemas titulado Turtle Island, que salió a la luz en 1974.

'Manzanita' comprende 18 poemas de los cuales hemos analizado los que figuran bajo la denominación de 'Anasazi', 'The Way West, Underground', 'Without', 'The Dead by the Side of the Road' $y$ 'Manzanita'.

Para la estructuración del análisis de los poemas en 'Manzanita' tenemos en cuenta un macrocontexto clave, distintivo de la idea global bajo la cual se agrupan todos los poemas comprendidos en la denominación genérica de 'Turtle Island', y que, como el poeta indica, es una frase-símbolo explicativa de la idea engendrada por la creencia milena- 
ria de que el universo, así como la tierra, están sostenidos por una gran tortuga o serpiente con atributos eternos. Tal concepción, en una versión más localizada, se atribuye también al continente americano en general, y a Norteamérica en particular. En el macrocontexto clave tenemos, pues, los tres ingredientes básicos de espacio, tiempo y movimiento. El tiempo, en sí mismo, presenta un espacio continuo = eternidad.

Otro aspecto estructural en el análisis de los poemas en 'Manzanita' es el submacrocontexto clave que distingue una idea como parte integrante de la idea global, y que es aplicable a la denominación de 'Manzanita'. Si 'Turtle Island' propone una concepción cosmológica con una implicación de solidaridad entre los humanos, 'Manzanita' es una llamada a la naturaleza, a lo genuino, a la espontaneidad representada en el árbol, donde se destaca el fruto encarnado que brota de su alma libre y salvaje. Tenemos, pues, el universo, la tierra, y 'Manzanita', como manifestación cósmica y microcósmica, pero todo formando un concierto armónico que interpreta la melodía de la aventura vital.

En la trama estructural, el microcontexto clave distingue una idea en forma de poema que está encuadrada en el submacrocontexto clave de 'Manzanita'. Tal idea puede tener un componente subjetivo sin una situación específica que le sirva de soporte espacial, un componente objetivo que se conjuga con una situación determinada, y donde se manifiesta un propósito estético por parte del poeta, o puede tener una combinación de ambos componentes, sirviendo el segundo como generador del primero, de forma que el objeto suscita la idea en el sujeto.

Con el epígrafe de macrocontexto ideologico queremos hacer resaltar cuál es la idea matriz en el contexto del poema, y con el de macroidea cuál es la conexión que el macrocontexto ideológico tiene en relación con la concepción dual de vida/muerte.

El macroespacio semántico, dentro del macrocontexto ideológico, establece las características de la situación o marco en el que la idea se encuadra en el poema, poniendo de manifiesto su naturaleza subjetiva, objetiva o comparativa, según sea un producto del sujeto, un reflejo de la realidad circundante o un resultado comparativo debido al poder de evocación del objeto sobre el sujeto (el poeta).

En el macroespacio semántico distinguimos un microespacio semántico que resalta cuáles son los tipos de frases que el poeta utiliza para expresar el contenido del macrocontexto ideológico (circunstancia o suceso, orden o petición, hipótesis, duda, deseo, con las posibles variantes modales de aseveración, negación y pregunta).

El submicroespacio semántico engloba los términos léxicos que el macroespacio semántico distingue para la expresión del macrocontexto ideológico. Los términos léxicos pueden dividirse, según el aspecto se- 
mántico de su contenido, en términos estáticos, dinámicos, simbólicos y representativos. La división en términos estáticos y dinámicos, a los que podemos denominar, de forma global, como términos referenciales, está relacionada con la idea de movimiento, en cuanto que su contenido indica una capacidad de acción o falta de ella (a tiger, a stone). $\mathrm{El}$ principio de movimiento está implícitamente intrínseco en el contenido semántico de los términos estáticos por la naturaleza rotatoria del sistema solar. Los términos simbólicos son términos representativos de carácter paradigmático. Los términos representativos son aquellos cuyo contenido se toma como representativo de toda una especie (man), de un grupo (flock) o de un ente determinado (Charles, Rome).

Los términos léxicos se clasifican, a su vez, en objetivos y subjetivos, de acuerdo con la capacidad de que su postulado sea susceptible de ser percibido por los sentidos o no lo sea. Los términos léxicos de carácter objetivo pueden ser estáticos o dinámicos, pero los de naturaleza subjetiva no son clasificables en esta categoría, asimismo los primeros están relacionados con los conceptos de movimiento y espacio, mientras que los segundos carecen de estas coordenadas, ya que son un producto del sujeto, basado en unos parámetros que no tienen asiento en el mundo exterior al mismo.

El contenido de los términos léxicos define su función en dos grandes apartados: elementos determinantes (verbo) y determinados. Dentro de los elementos determinados están los determinados por el verbo, de carácter primario y secundario (sujeto, objeto) (éste como complemento del significado verbal), o son indicativos de una circunstancia que completa o extiende el significado del determinante. Tal circunstancia puede ser indicativa de un espacio, tiempo o modo (he came to the station, at four o'clock, with his sister). Una circunstancia es también atribuible al nombre (the power of money $=$ indicativo de identidad).

El contenido de los elementos determinados y determinantes se altera por medio de términos modificadores (adverbios) y determinantes (adjetivos), respectivamente; el adverbio es, a su vez, un término modificador del adjetivo.

La función determinante tiene un carácter dinámico, mientras que los elementos y términos determinados se caracterizan por su naturaleza estática.

Otras de las funciones de ciertos vocablos es la de representar o relacionar, teniendo ambas un cometido formal sin que exista una implicación semántica, en cuanto que la representación o la relación estén basadas en el significado (they, and). De acuerdo con lo apuntado anteriormente sobre los términos representativos, éstos pueden tener una función semántica o un cometido puramente formal. En relación 
con los nombres propios, la representación que ostentan es de carácter distintivo, pudiendo, a su vez, tener los atributos de términos dinámicos o estáticos (Mary, Everest).

Hemos creído conveniente realizar esta prolongada explicación relativa a la estructuración del submicroespacio semántico, ya que en el análisis de los poemas encuadrados en el submacrocontexto clave de 'Manzanita', vamos a utilizar la terminología concerniente a dicha estructuración.

Como es de suponer el microespacio semántico tiene correlación con un microespacio sintáctico que define un tipo de oración o frase, así como el submicroespacio semántico se corresponde con un submicroespacio sintáctico que comprende los distintos elementos oracionales, junto con la función que desarrollan en el contexto de la oración. Como es de suponer, en el análisis del submicroespacio semántico se dan connotaciones propias del submicroespacio sintáctico.

1. Pasando al estudio de los poemas de Gary Snyder en el macrocontexto clave 'Turtle Island' y el submacrocontexto clave 'Manzanita', nos encontramos con el microcontexto clave de Anasazi; de los términos simbólicos que configuran la expresión 'Turtle Island', de carácter subjetivo, vamos al término referencial de carácter estático 'Manzanita', para llegar a un término representativo de un entorno geográfico (Anasazi).

EI macrocontexto ideológico plantea una concepción panteísta: desde la tierra se llega a los dioses que la fecundan. La macroidea que subyace en el macrocontexto ideológico de 'Anasazi', en cuanto a la dualidad de vida/muerte, es la modalidad de la vida, que se identifica con la fertilidad de la tierra, y de la mujer en su capacidad de engendrar hijos, así como con el poder vitalizador del agua (trickling streams in hidden canyons/ under the cold rolling desert). A la macroidea del triunfo de la vida sobre la muerte se llega a través de un proceso comparativo que parte de la observación objetiva de un entorno geográfico (Anasazi), y conduce al sentir reflexivo del poeta; del objeto se va hasta el sujeto.

El macroespacio semántico del macrocontexto ideológico conforma una situación que tiene un enclave espacial en 'Anasazi', que constituye el objeto del poema. El macroespacio semántico está, pues, implícito en el microcontexto clave.

El microespacio semántico representativo del macrocontexto ideológico está configurado en los versos: 'sinking deeper and deeper in earth/ up to your hips in Gods\%. Se trata de una aseveración de carácter alegórico y matiz comparativo que configura el concepto de movimiento. 
El léxico que está bajo la demarcación del submicroespacio semántico está formado por un término dinámico (sinking), una expresión de contenido dinámico (deeper and deeper), un término estático (earth) (su contenido referencial es estático en relación con un sujeto, 'man'), términos simbólicos (hips, Gods), término determinante, indicativo de identidad (your) y términos de relación o conectores (and, in, up, to).

Funcionalmente hay un término, fuera del contexto de la frase, que forma parte del microespacio semántico, al que se le atribuye la misión de determinado (Anasazi), agente del fenómeno indicado por el elemento determinante 'sinking'; elementos determinados que son utilizados como indicativos de circunstancias, a manera de extensión del significado del elemento determinante, son 'earth', 'hips' y 'Gods', los tres de carácter espacial. Tenemos, pues, la combinación de movimiento/espacio, y la de objetivo/subjetivo, representado este último aspecto por los términos simbólicos 'hips' y 'Gods', y el aspecto objetivo por 'earth'. 'Deeper' es un elemento modificador del contenido del elemento determinante, que repetido, pone de manifiesto el concepto de moción. La función de determinar, y la formal de relacionar, están realizadas por 'your', en primer lugar, y por las diversas partículas en segundo lugar.

La idea de la fecundidad es un proceso motriz que necesita de un agente (Gods) y un objeto (Anasazi) indicador del espacio fecundado; 'earth' y 'hips' son parte integrante de 'Anasazi'.

Como se puede apreciar, el macroespacio semántico, explicativo del macrocontexto ideológico, que tiene su contrapartida en el macroespacio sintáctico, no refleja la normativa estructural, en el sentido de darse una alteración en la función que los términos léxicos realizan (Gods = elemento de carácter impulsor o agente es considerado como una circunstancia explicativa del elemento determinante 'sinking', pero son los dioses los que fecundan la tierra en el enclave de Anasazi. Por el contrario la correspondencia semántica entre términos léxicos y los conceptos espacio/movimiento, así como el aspecto modal objetivo/ subjetivo tiene una identidad plena.

2. El microcontexto clave The Way West, Underground define al poema que tiene como macrocontexto clave 'Turtle Island', y como submacrocontexto clave 'Manzanita'. El macrocontexto ideológico del poema está implícito en el microcontexto clave: el camino del este al poema está implícito en el microcontex civilización que representa el
oeste conduce a Europa, índice de una coundan
oscurantismo (underground) en comparación con las leyes que regulan la naturaleza. En el microcontexto clave, al igual que sucedía en 'Anasazi', hay una referencia a un espacio geográfico, esta vez sin una situación específica, a lo que se añade una indicación de movimiento. 
La macroidea que encierra el macrocontexto ideológico presenta una alternativa en la combinación de los conceptos vida/muerte, ya que la civilización, representada por países del este terráqueo tiene su contrapartida en la falta de civilización en el oeste (Europa), en donde países como Francia, y en especial España, conocen las sombras de la noche.

El macroespacio semántico del macrocontexto ideológico muestra la ausencia de una situación definida, puesto que diversos destellos de una máquina fotográfica imaginable ponen de relieve lugares como 'Oregon', 'North Japan', 'China', 'Tibet', 'Finland', 'France' y 'Spain', que se encuentran en el mundo del ensueño (Oregon), o en el camino del este al oeste.

El mismo signo negativo que se aprecia en el macroespacio semántico con respecto a una situación, se deja ver en el microespacio semántico en relación con una frase cuyo contenido se tome como representativo del macrocontexto ideológico, ya que son diversos términos léxicos, propios del submicroespacio semántico, los que conforman el macrocontexto ideológico. Términos dinámicos tales como 'moves', 'fly' y 'race' son utilizados como indicadores de un movimiento rotatorio. Términos representativos de espacios son aquellos que se utilizan para denominar continentes (Europa) o países (Finland). Los términos simbólicos (bear, underground) forman parte del mundo de la subjetividad, mientras que los términos representativos de lugares, aunque su poder de referencia puede ser constatado en el mundo real, sin embargo, al no ser indicadores de una situación determinada, definen el contenido del poema como de carácter subjetivo, encerrándose en el mismo la intencionalidad ideológica del poeta.

De la lectura de 'Anasazi' y 'The Way West, Underground' se infiere la constante que domina el mundo afectivo/ideológico de Gary Snyder, entrenado en los postulados fisicomentales de Zent, y que elige los espacios abiertos que circundan las montañas californianas, como santuario y vivienda; la mente, el espíritu necesita de la naturale$\mathrm{za}$, como el agua de la fuente, para conocer su origen e identidad. El poeta, heredero a ultranza de un maniqueísmo programado, divide el mundo de los humanos en dos mitades irreconciliables: lo natural y lo artificial; lo civilizado y lo no civilizado. Los países y regiones del mundo son objeto de esta diferenciación según estén más cerca o más lejos de un ideal en donde los hilos del alma tañen con ritmo sereno la melodía del universo; la violencia es la fuerza incontrolada que convierte dicha melodía en una sucesión de ruidos ensordecedores.

3. Dentro del mismo macrocontexto y submacrocontexto clave, que en los dos poemas anteriormente citados, el microcontexto clave Without distingue en su macrocontexto ideológico a la naturaleza como 
fuente de energía, y define la existencia dentro de unas coordenadas de movimiento (actividad) y de tiempo (el presente), confiriendo a la misma un sentido lúdico (the end is,/ grace-ease-/), observándose una velada crítica a un tipo de religión oficialista (healing,/ not saving./).

Dominando las diversas microideas que configuran el credo vital de Snyder, se destaca el concepto de 'vida' en la conjunción de la macroidea vida/muerte.

El macroespacio semántico del macrocontexto ideológico se distingue por la ausencia de una situación que lo defina, poniéndose así de manifiesto el carácter subjetivo del contenido del poema.

El microespacio semántico que concretiza el macrocontexto ideológico, se manifiesta por medio de 'the power within./ the power/without'./ (frases aseverativas de naturaleza subjetiva que expresan la idea implícita en el microcontexto clave).

El submicroespacio semántico está formado por un término subjetivo, repetido por dos veces (power), con una función de elemento determinado primario (en contraposición a uno secundario = objeto, circunstancia), que se ve privado del elemento determinante (is). La naturaleza estática del contenido de 'is' hace que el elemento determinado deje de ser un elemento agente. Los términos 'within' y 'without' son elementos modificadores del determinante (is). Estos dos vocablos son clave para la expresión ideológica del poeta, que de nuevo se sirve de un contraste, y que también utiliza de nuevo una alusión espacial, pero en este caso el espacio es el que abarca el ser humano, que trasciende el cuerpo del hombre, y el espacio del universo donde se engendra la vida. El movimiento en forma de rotación se da de fuera a dentro: la naturaleza confiere poder, energía al ser humano; la parte dependiente del todo.

Es de advertir cómo el carácter subjetivo del poema se manifiesta por la ausencia de una situación determinada, y por el uso de términos subjetivos (power).

4. El microcontexto clave The Dead by the Side of the Road implica el macrocontexto ideológico del poema, en el que a través de la anécdota, que tiene un espacio específico (the road), se expresa la dualidad reiterativa entre civismo y falta del mismo; el primero radica en una atención preferente por la naturaleza, pero la mecanización, producto del progreso, destruye la vida animal en la carretera, y el hombre utiliza a los animales para sus propios fines. La macroidea en el trasfondo del poema indica la alternativa de la destrucción, la muerte, frente a la vida.

El macroespacio semántico del macrocontexto ideológico se exterioriza a través de una situación objetiva, que tiene como protagonista 
la carretèra, y está formada por escenas recurrentes, como aspectos de un mismo cuadro pictórico.

El microespacio semántico de (4) coincide con el microcontexto clave: 'the dead by the side of the road'. Se trata de una aseveración en forma de circunstancia o suceso.

El submicroespacio semántico comprende los términos estáticos 'side' y 'road', siendo el primero un término definitorio de un espacio (road). 'Dead' es un término subjetivo de naturaleza negativa, aplicable a la falta de movimiento, como contrapartida de la vida. Funcionalmente el elemento determinante, no explícito (is), tiene como elemento determinado a 'dead', con capacidad de existencia, y como elemento determinado indicativo de una circunstancia a 'side'; 'road' es un elemento determinado que expresa una circunstancia relativa a 'side'. La función de relación la llevan a cabo los términos 'by' y 'of'. 'The' es un término señalizador.

Gary Snyder denuncia en (4) la lucha que el hombre sostiene con la vida animal, la naturaleza, triunfando la ley del más fuerte en recursos de capacidad creadora; la voz del juglar quiere hacer llegar su eco al oído del lector para recordarle que es parte de la naturaleza, y si la destruye, se destruye a sí mismo, destrozando el vínculo que le une a la vida.

5. Dentro del macrocontexto clave de 'Turtle Island', se da una coincidencia entre el submacrocontexto y el microcontexto clave en el término léxico de Manzanita. El poema expone una situación a la que falta un macrocontexto ideológico, destacándose ciertos aspectos representativos entre los que se encuentran el coyote como símbolo de libertad; un tiempo, el de la puesta de la luna; un animal, el perro; un ave, el pajaro carpintero; un hombre que destruye, y el fruto del acerolo, símbolo de lo natural, lo salvaje.

El macroespacio semántico es el contexto de una situación de carácter objetivo, indicativa de un espacio, el campo, donde la retina del observador vibra ante los destellos irisados de la vida.

Como no se da un macrocontexto ideológico en (5), no se obtiene un microespacio semántico que lo pueda interpretar, y solamente se observan descripciones, de las cuales la más interesante es la de la manzanita, el fruto del acerolo (Manzanita de tips in fruit,/ Clusters of hard green berries/ The longer you look/ The bigger they seem,/ "little apples"/). Parece existir una referencia al árbol, cuando se utiliza el término 'Manzanita'.

Gary Snyder se deleita en la transmisión de percepciones que tienen lugar en el ámbito ideal de la naturaleza, en el momento del despertar, poniendo en un primer plano fotográfico al acerolo que engen- 
dra su fruto, y mezclando la situación dentro de una gama de colores (gold-purple, gray, red, green).

El análisis de los poemas en el macrocontexto clave 'Turtle Island' y submacrocontexto clave 'Manzanita' pone de manifiesto algunas constantes, relacionadas con las distintas formas estructurales que hemos tratado.

Las constantes del macrocontexto ideológico apuntan a valorar la naturaleza como fuente de vida y marco de placer estético. La macroidea que aflora en las aguas de la idea destaca una constante, en la que se establece el triunfo de la vida sobre la muerte.

El macroespacio semántico muestra una continuidad en la exposición de situaciones dentro de un espacio, en las que a través del objeto, y siguiendo un proceso de apreciación comparativa, el sujeto (el poeta) llega a formular sus reflexiones.

El microespacio semántico de los diversos poemas muestra una tendencia al uso de frases aseverativas de carácter subjetivo, cuando se da un macrocontexto ideológico, y en consecuencia, la necesidad de expresarlo. Se entiende por frase subjetiva aquella que no refleja el mundo exterior al sujeto.

En relación con las expresiones de tiempo, espacio y movimiento, hay una mayor incidencia en las indicaciones de espacio.

Los distintos submicroespacios semánticos muestran una prioridad en el uso de términos léxicos de carácter subjetivo, lo que se corresponde con la preferencia del poeta por expresar sus ideas por medio de frases aseverativas del mismo carácter subjetivo; los términos de contenido estático y dinámico, así como los simbólicos les siguen en preferencia de uso.

Las funciones de los diversos términos léxicos completan el contexto del submicroespacio semántico, pudiendo observarse un predominio de los términos que realizan la función formal de relacionar, seguidos de los que expresan circunstancias del determinante, y de los que lo modifican, a los que se añaden los que tienen el cometido de determinar (the). En la relación elemento determinante/determinado se hace notar una predisposición a la omisión del primero.

Hemos esbozado, de forma genérica, la posibilidad de analizar un poema, tomado como medio lingüístico de expresión de una idea. A continuación vamos a mostrar la puesta en práctica, de forma detallada, del esquema de análisis propuesto, examinando el poema de Gary Snyder que figura con el microcontexto clave 'As for Poets', dentro del submacrocontexto clave 'For the Children' y el macrocontexto clave 'Turtle Island'.

El microcontexto clave distingue una idea global en la que se implica la identificación del concepto de 'poeta', con las manifestaciones de los componentes de la naturaleza, de forma que el objeto suscita 
comparaciones a la manera de alegorías en la mente del sujeto (el poeta).

El macrocontexto ideológico destaca el principio de que la poesía es obra de poetas como la tierra (Earth Poets), el aire (Air Poets), el fuego (Fire Poets), el agua (Water Poet), el espacio (Space Poet) y la mente (Mind Poet), haciéndose notar la singularidad del agua, el espacio y la mente como elementos creadores.

La macroidea que encierre el poema pone énfasis en el aspecto vital, en contraposición con el aspecto negativo de la muerte.

El macroespacio semántico conforma una situación subjetiva donde el poeta, teniendo como eje la tierra, el aire, el fuego, el agua, el espacio y la mente, valora la actividad de los mismos como labor de poetas creadores de belleza.

El microespacio semántico que resume el macrocontexto ideológico es 'as for poets', que coincide con el microcontexto clave. Se trata de una frase que se completa en cada una de las circunstancias expresadas en las distintas estrofas de que se compone el poema.

El submicroespacio semántico, que el macroespacio semántico distingue para la expresión del macrocontexto ideológico, está formado por dos términos de relación (as for) y un término simbólico (poets). Hay una ausencia de elemento determinante, así como del resto de la frase que se continúa en cada una de las estrofas. Otra lectura de la frase 'as for poets' puede ser 'if it is concerned with poets...'

Siguiendo la idea pendular de que el hombre es parte de la naturaleza, con la que constituye una unidad de sentido, Gary Snyder sublima los componentes vitales de la misma.

Dentro del macrocontexto ideológico del poema, podemos considerar seis submacrocontextos ideológicos que se corresponden con sus seis estrofas, y que tienen como sujeto a los diversos componentes de la madre naturaleza. En el aspecto formal la estrofa $l$ tiene un marco de exposición de cuatro versos; la 2 cinco versos; la 3 seis versos y la 4,5 y 6 ocho versos cada una, formando un total de treinta y nueve versos.

ESTROFA N $\mathbf{N}^{\circ} 1$

En esta estrofa se aprecia un submicrocontexto clave en el que se da una situación genérica, donde el objeto (the earth) suscita en la mente del poeta una comparación de naturaleza alegórica. El submacrocontexto ideológico comporta la concepción de la tierra como un 
poeta, cuyas manifestaciones son poemas, sin igual entre los que los hombres elaboran. El submacrocontexto semántico configura una situación subjetiva que sirve como marco a la inspiración del poeta, que usa a la tierra como eje comparativo. El submicroespacio semántico está integrado por una frase alegórica en forma de circunstancia aseverativa que va precedida de la frase introductora 'as for poets', que repite el microcontexto clave (As for poets/The Earth Poets/Who write small poems,/Need help from no $\mathrm{man} /$.).

El concepto de tiempo tiene su expresión por medio del verbo (need) que indica un presente genérico abarcando el antes, el ahora y el después $(-0+0)$, y el espacio está representado por 'earth'.

El sub-submicroespacio semántico tiene la siguiente lectura léxica de términos y sus funciones:

a) Frase introductora, ya analizada (As for poets).

b) (The earth poets who write small poems, need help from no man).

1) Elemento determinado primario,

2) elemento determinante,

3) elemento determinado secundario (objeto), considerado como una extensión de significado al determinante,

4) extensión de significado al determinante en forma de circunstancia indicativa de identidad.

1) Comprende un término simbólico (poets), determinado por un término de identificación (earth) = dinámico (relativo a), objetivo (sólido), y por uno señalizador de naturaleza anafórica (the), todo lo cual está, a su vez, determinado por la frase adjetiva que tiene como elemento determinado un término representativo (who), y como determinante a 'write' $(-0+0)$ $=$ término simbólico, al que sigue un elemento determinado secundario (poems) = término simbólico que está, asimismo, determinado por un término de naturaleza subjetiva (small).

2) Consta de 'need' = término subjetivo $(-0+0=$ presente genérico).

3) Se compone de 'help' = término subjetivo.

4) Consta de 'man' = término dinámico, objetivo, de carácter representativo, que se encuentra determinado por 'no'; como elemento de relación entre 'help' y 'man' se halla 'from'.

Al analizar un poema detalladamente, creemos conveniente indicar el patrón rítmico que corresponde a cada estrofa, basado en la periodicidad del acento. Ello está fuera del esquema de análisis pro- 
puesto en el análisis general. El patrón rítmico correspondiente a la

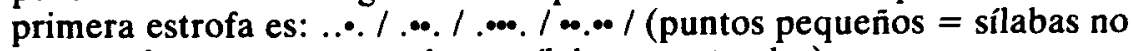
acentuadas y puntos grandes $=$ sílabas acentuadas).

ESTROFA N $\mathbf{N}^{\circ} 2$

El submicrocontexto clave, en el contexto de la estrofa, destaca una situación genérica, con una substancia (the air) y una comparación alegórica por parte del sujeto (el poeta). (The air Poets / Play out the swiftest gales/ And sometimes loll in the eddies/. Poem after Poem,/ Curling back on the same thrust/).

El submacrocontexto ideológico encierra la idea del aire como poeta excelso, canta-autor de poemas, mientras que el submacroespacio semántico implica una situación subjetiva en la que, comparativamente, el poeta considera el aire como creador de poesía.

El submicroespacio semántico, que expresa el contenido del submacrocontexto ideológico, está formado por tres alegorías en forma de circunstancias aseverativas:

a) (The air poets play out the swiftest gales).

b) (And sometimes loll in the eddies).

c) (Poem after poem, curling back on the same thrust).

El concepto de tiempo se manifiesta en 'sometimes' y en el contenido verbal $(-0+0=$ presente genérico). La idea de movimiento tiene su expresión en forma de repetición (poem after poem), así como en el contenido verbal 'curling back'.

El sub-submicroespacio semántico aplicable a los términos léxicos que el submacroespacio semántico distingue para la expresión del submacrocontexto ideológico, está integrado por los siguientes elementos y términos léxicos en cada una de las tres frases que componen la estrofa:

a) (The air poets play out the swiftest gales).

1) Elemento determinado primario (sujeto),

2) elemento determinante,

3) elemento determinado secundario (objeto).

1) Está constituido por un término simbólico (poets), determi- 
nado por un término señalizador con referencia anafórica (the), y precedido del término de identificación (air) = dinámico, objetivo (gas).

2) Consta de un término simbólico (play), indicativo de una acción generalizada $(-0+o)$ al que modifica 'out'.

3) Está formado por 'gales' = término simbólico que está determinado por 'swiftest' = término subjetivo de matiz comparativo al que acompaña 'the', con carácter señalizador y anafórico.

b) (And sometimes loll in the eddies).

1) Elemento determinado primario omitido (the aire poets),

2) elemento determinante,

3) extensión de significado en forma de circunstancia indicativa de espacio. 'And' es un elemento de relación que une $a$ con $b$.

2) Comprende un término simbólico (loll), indicativo de una actividad generalizada, a quien modifica 'sometimes', expresión de un tiempo indefinido.

3) Consta de un término simbólico (eddies) que está relacionado con el determinante por medio de 'in', y determinado por 'the'.

c) (Poem after poem, curling back on the same thrust).

1) Elemento determinado primario omitido (the air poets),

2) elemento determinante, con 'are' omitido,

3) elemento determinado secundario,

4) extensión de significado en forma de circunstancia indicativa de un espacio. La lectura de la frase, con la inclusión de los términos omitidos es: ('The air poets) (are) curling back poem after poem on the same thrust'.

2) Comprende el término simbólico '(are) curling' = dinámico, indicativo de una acción generalizada, al que modifica 'back'.

3) Está constituido por un término simbólico (poem) que se repite, teniendo como término de relación a 'after'.

4) Comprende un término simbólico (thrust) que se halla relacionado con el determinante por 'on', y determinado por 'same' = término subjetivo, indicativo de identidad, además de sufrir la determinación de 'the'.

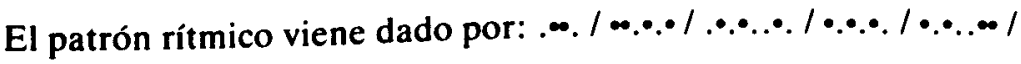




\section{ESTROFA No 3}

En el submicrocontexto clave se observa un componente objetivo que sirve de soporte al poeta para, de manera comparativa, llegar a una apreciación de carácter subjetivo. (At fifty below/ Fuel oil won't flow/ And propane stays in the tank/. Fire Poets/ Burn at absolute zero/ Fossil love pumped back up/.)

El submacrocontexto ideológico expone la idea de que a temperaturas gélidas, donde la naturaleza parece muerta, se detecta la existencia de poetas ígneos (Fire Poets) que reciben su calor del amor engendrado en la tierra.

El submacroespacio semántico implica una situación subjetiva en la que, comparativamente, el poeta considera que aún donde el hielo detiene el pulso de la naturaleza el calor creador alienta.

El submicroespacio semántico está integrado por cuatro frases: a) (At fifty below fuel oil won't flow). b) (And propane stays in the tank). c) (Fire poets burn at absolute zero). d) (Fossil love pumped back up). $a$ y $b$ son dos condiciones (hipótesis) indicativas de leyes naturales; la primera planteada de forma negativa y la segunda afirmativa, y $c$ y $d$ son dos alegorías en forma de circunstancias aseverativas. $a$ y $b$ establecen un contraste con respecto a $c, y c$ es el efecto que tiene como causa a $d$ (if we are at fifty below, fuel oil won't flow and propane stays in the tank, but even if we were at absolute zero, fire poets would burn because fossil love is pumped back up).

El concepto de tiempo viene indicado por medio del verbo que expresa dos acciones, un proceso y. un estado habitual $(-0+0)$; en $a$ y $b$ de carácter universal, aunque el tiempo verbal sea futuro en 'flow'; en $d$ 'is' está omitido. El espacio tiene su representación en 'tank' y el movimiento en '(is) pumped'.

El sub-submicroespacio semántico está formado por los siguientes elementos, junto con los términos léxicos que integran las diversas frases:

a) (At fifty below fuel oil won't flow) que tiene la interpretación de 'if we are at fifty below, fuel oil won't flow', o 'when we are...' En el antecedente 1) elemento determinado primario omitido, 2) elemento determinante omitido, 3) extensión de significado en forma de circunstancia indicativa de modo, 4) extensión de significado secundaria (a un nombre). En el consecuente 1) elemento determinado primario, 2) elemento determinante. En el antecedente el conector 'if' está omitido. 3) Consta de un elemento determinado que está omitido (degree), y relacionado con el determinante por 'at', a la vez que deter- 
minado por el término 'fifty' = cuantificador de carácter objetivo. 4) Comprende el término de relación 'below' que une a 'fifty' con el término omitido 'zero'. En el consecuente 1) está formado por 'oil' = término dinámico, objetivo (líquido), que se encuentra precedido del término de identificación 'fuel' = término de naturaleza objetiva. 2) Está integrado por 'flow' = proceso ( $+o=$ futuro), al que 'not' modifica.

b) Esta frase está unida con $a$ a través de un elemento de relación (and) = conector conjuntivo. (And propane stays in the tank.) 1) Elemento determinado primario, 2) elemento determinante, 3) extensión de significado en forma de circunstancia indicativa de espacio. 1) Consta de un término dinámico, objetivo de componente gaseoso (propane). 2) Está constituido por un término estático (stays), (o = presente). 3) Comprende un término estático, objetivo, de componente sólido (tank), elemento determinado secundario que está relacionado con el determinante por medio de 'in'. El término señalizador de carácter anaforico (the) determina a 'tank'.

c) (Fire poets burn at absolute zero.) 1) Elemento determinado primario, 2) elemento determinante, 3) extensión de significado en forma de circunstancia indicativa de modo o manera. 1) Está formado por un término simbólico (poets), al que precede un término identificativo (fire) = término dinámico, objetivo, de componente gaseoso. 2) Comprende un término simbólico (burn), ( $-0+0=$ presente genérico). 3) Se compone del elemento determinado secundario 'zero', de naturaleza simbolica, que está determinado por un término subjetivo, de matiz enfático (absolute), y relacionado con el elemento determinante por medio de 'at'.

d) (Fossil love pumped back up.) 1) Elemento determinado primario, 2) elemento determinante. 1) Se compone de un término simbólico (love) que está determinado por un término subjetivo (fossil). 2) Está integrado por '(is) pumped', en donde el primer término está omitido, configurando una forma estructural estilística (voz pasiva), de matiz estático, cuya naturaleza es simbólica. El tiempo implícito es un presente genérico ( -0 $+o)$. Este elemento determinante está modificado por 'back' y 'up', indicativos de dirección hacia unos espacios.

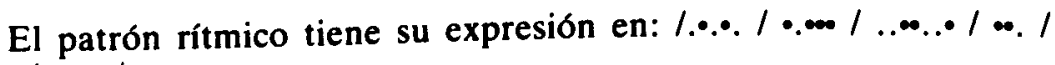
$\cdot . \cdot . \cdot \cdot / \cdot .000 /$ 
El submicrocontexto clave detecta un componente subjetivo que comporta una situación de carácter alegórico que implica un componente objetivo, como es el origen de la vida a través del agua; este principio biológico da lugar, de forma comparativa, a una ensoñación subjetiva por parte del poeta. (The first/ Water Poet/ Stayed down six years/. He was covered with seaweed/. The life in his poem/ Left millions of tiny/ Different tracks/ Criss-crossing through the mud/.)

En el submacrocontexto ideológico se expresa la idea del agua como poeta, que después de estar sumergido durante seis años y cubierto de algas, creó un poema que encerraba la vida.

El submacroespacio semántico implica una situación subjetiva con un substrato histórico-geológico y bíblico.

El submicroespacio semántico, que expresa el contenido de submacrocontexto ideológico, está formado por tres frases:

a) (The first water poet stayed down six years).

b) (He was covered with seaweed).

c) (The life in his poem left millions of tiny different tracks crisscrossing through the mud).

Las tres frases son alegorías en forma de circunstancias aseverativas. $b$ se manifiesta como una consecuencia de $a$ : (The first water poet stayet down six years so he was covered with seaweed).

El concepto de tiempo viene expresado a través del contenido verbal $(-0=$ pasado). Tales contenidos verbales están relacionados con estados (stay, was covered) y con acciones (left, [were] criss-crossing). Una indicación de tiempo extrínseca al tiempo verbal es 'six years'. La apreciación de espacio se identifica con el modificador "down' y con la expresión "through the mud'. El concepto de movimiento se manifiesta en '(were) criss-crossing'.

El sub-submicroespacio semántico está formado por los siguientes términos léxicos, dentro de los elementos que integran cada una de las tres frases que forman el submicroespacio semántico:

a) (The first water poet stayed down six years.) 1) elemento determinado primario, 2) elemento determinante, 3) extensión de significado indicativa de tiempo. 1) Comprende un término simbólico (poet) al que acompaña 'water' = término de identificación, dínámico, objetivo, de componente líquido, siendo 
éste determinado por 'first' = término subjetivo, cuantificador de matiz temporal, que implica una división del tiempo en espacios. 2) Consta de 'stayed' = estático, $(-0)$, con un modificador que implica una situación con respecto a un espacio (down). 3) Se compone de un elemento determinado secundario (years) = término simbólico, que se ve determinado por el término simbólico 'six'.

b) (He was covered with seaweed.) 1) Elemento determinado primario, 2) elemento determinante, 3) extensión de significado (circunstancia). 1) Está integrado por un término representativo (he). 2) Se forma con un término estático (voz pasiva), $(-0)$ (was covered). 3) Consta del elemento determinado secundario (seaweed) = término simbólico, que está unido al determinante por un término de relación (with).

c) (The life in his poem left millions of tiny different tracks, crisscrossing through the mud.) 1) Elemento determinado primario, 2) extensión de significado secundaria con una configuración espacial, 3) elemento determinante, 4) elemento determinado secundario (objeto), 5) extensión de significado secundaria de carácter identificativo. 1) Comprende un término dinámico, conectado con el movimiento, de índole universal (life), que está determinado por un término señalizador de referencia anafórica (the). 2) Está formado de un término simbólico (poem) que tiene conexión con el elemento determinado primario por medio de 'in', y se encuentra determinado por un término indicativo de identidad (his). 3) Comprende un término dinámico (left), (-o). 4) Se compone del elemento determinado (millions) = cuantificador de matiz genérico. 5) Consta de un término simbólico (tracks) al que determina un término subjetivo (different) $=$ distinción de identidad, y éste, a su vez, se ve determinado por un término subjetivo 'tiny'. 'Tracks' está conectado con el elemento determinado secundario a través del término de relación 'of'. Otro determinante de 'tracks' es la frase descriptiva (criss-crossing through the mud), en donde 'that' está omitido, y consta de un elemento determinante '(were) criss-crossing' = término dinámico, $(-0)$, con una extensión de significado en forma de circunstancia indicativa de un espacio, integrada por un término simbólico (mud), que se encuentra relacionado con el elemento determinante por medio de 'through', y determinado por 'the'. El elemento determinado primario es 'tracks', representado por 'that', si estuviera explícito. 


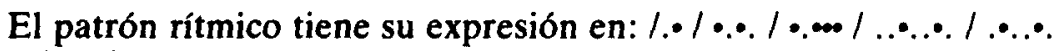
$|\bullet . . . \cdot| \cdot \ldots / \cdot \ldots . . \% \mid$

ESTROFA N $\mathbf{N}^{\circ} 5$

El submicrocontexto clave tiene un componente subjetivo que comporta una situación alegórica, partiendo del principio del espacio sideral como infinito, aunque, paradojicamente, se le pueda añadir una línea divisoria. El espacio, como concepto de relación entre cuerpos solidos, es la fuente de inspiracion del poeta. (With the Sun and the Moon/In his belly,/ The Space Poet/ Sleeps./ No end to the sky-/ But his poems, / Like wild geese,/ Fly off the edge/.)

El submacrocontexto ideológico encierra la idea del espacio como un poeta, que cohabita con el sol y la luna, dormitando en una ausencia de movimiento, pero en su recinto sin límites crea belleza que desborda su propio entorno.

El submacroespacio semántico implica una situación subjetiva que, marginalmente, alude al problema filosófico-matemático de la concepción de 'infinito' aplicado al espacio sideral.

El submicroespacio semántico está constituido por tres frases:

a) (With the sun and the moon in his belly, the space poet sleeps).

b) (No end to the sky).

c) (But his poems, like wild geese, fly off the edge).

$a$ y $c$ son dos alegorías en forma de circunstancias aseverativas. En $a$ se da una transposición de espacios en la estructura de la frase, siendo la manera usual; (The space poet sleeps with the sun and the moon in his belly). El contenido de $b$ es el de una verdad aceptada como general, que expone un principio motivo de controversia. $b$ y $c$ establecen un contraste a manera de concesión; otra posible lectura es: (although [there is] no end to the sky, his poems, like wild geese, fly off the edge).

El concepto de tiempo tiene su expresión en la formal verbal ( - o $+o)=$ acontecer generalizado. La expresión léxica de espacio se realiza por medio de 'space', y por 'end' y 'edge', que implican (end of something) y (edge of something), asi como por 'off' (on the other side of). La idea de movimiento se manifiesta en 'fly'.

El sub-submicroespacio semántico está integrado por los siguien- 
tes elementos funcionales y términos léxicos en cada una de las tres frases:

a) (With the sun and the moon in his belly the space poet sleeps.)

1) Extensión de significado al elemento determinante en forma de circunstancia indicativa de modo, 2) extensión de significado secundaria, indicativa de espacio, 3) elemento determinado primario, 4) elemento determinante. 1) Se compone de los elementos determinados secundarios ('sun' y 'moon') = términos dinámicos, dentro de una dinámica sideral, objetivos (gaseoso y sólido), que están relacionados entre sí por medio de 'and', determinados por 'the', y puestos en contacto con el determinante por medio de 'with'. 2) Comprende el elemento determinado terciario (belly) = término simbólico que está determinado por el término de identificación 'his', y conectado con el elemento determinado secundario a través de 'in'. 3) Consta de un término simbólico (poet), al que acompaña un término de identificación (space) = subjetivo (relativo a entes objetivos). 4) Está integrado por un término simbólico, indicativo de un estado habitual $(-0+o)$ (sleeps).

b) '(There is) no end to the sky'. 1) Elemento determinado primario, omitido, 2) elemento determinante, omitido, 3) elemento determinado secundario que se considera como una extensión de significado primaria en calidad de objeto, 4) extensión de significado secundaria. 1) Está implícito 'there' = término de carácter anafórico indicativo de espacio. 2) Está implícito 'is' = término indicativo de existencia, $(-0+0=$ naturaleza permanente). 3) Comprende un término subjetivo implicativo de espacio (end), que se encuentra determinado por un término negativo (no). 4) Está formado de 'sky' = dinámico, objetivo (gaseoso), siendo 'to' un término de relación que lo une con el elemento determinado secundario, $y$ 'the' un término determinante.

c) (But his poems, like wild geese, fly off the edge.) 'But' es un elemento de relación con $b$ (partícula coordinadora $=$ contraste). 1) Elemento determinado primario, 2) elemento determinante, 3) extensión de significado. 1) Está formado por un término simbólico (poem), siendo determinado por un término de identificación (his), y por la frase 'like wild geese' (that are like wild geese), que tiene como determinado y determinante implícitos a 'that' $y$ 'are', respectivamente, (el primero término representativo, y el segundo con la lectura 'are considered'). 'Geese' = térmi- 
no 'simbólico, al que determina el término subjetivo 'wild', está relacionado con el determinado primario por medio de 'like', indicativo de comparación. 2) Consta de un término dinámico (fly), $(-0+0)=$ actividad genérica, modificado por 'off' que implica un espacio. 3) Está constituido por un término simbólico (edge), determinado por el término señalizador anafórico 'the'. $\infty . \bullet$

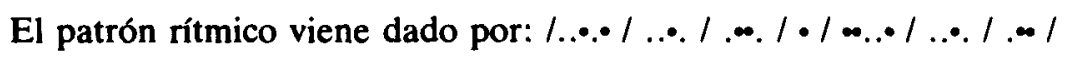

ESTROFA No 6

En el submicrocontexto clave se da un componente subjetivo que comporta una situación de carácter alegórico que parte del principio de la mente como marco de la vida. (A Mind Poet/ Stays in the house./ The house is empty/ And it has no walls./ The poem/Is seen from all sides/ Everywhere,/ At once/.)

El submacrocontexto ideológico contiene la idea de la mente como generadora de vida y creadora de belleza, siendo equiparable a una deidad.

El submacroespacio semántico implica una situación subjetiva, aludiendo al problema ontológico de la existencia de un ente razonador que regula el universo, y que se hace patente al observador.

El submicroespacio semántico, expresión del contenido del submacrocontexto ideológico está formado por cuatro frases: a) (A mind poet stays in the house), b) (The house is empty), c) ([And] it has no walls), d) (The poem is seen from all sides, everywhere, at once). $a$ y $d$ son dos frases alegóricas en forma de circunstancias aseverativas, $y$ $b$ y $c$ se caracterizan por ser dos frases descriptivas, circunstancias aseverativas de carácter alegórico, aplicables al término simbólico 'house'; estas dos últimas frases están en una relación de coordinación conjuntiva (and).

El concepto de tiempo se manifiesta en 'stays', 'is', 'has', - o +o (estado permanente con valor existencial), y en la expresión 'at once'. El espacio se indica con 'sides' (límites de un espacio), y con 'everywhere' de carácter universal. Hay dos contradicciones semánticas en la estrofa: la primera se refiere al hecho de que alguien esté en su casa, y a la vez esté vacía, y la segunda apunta al concepto de finito, implícito en 'all sides', y el de infinito en 'everywhere'. El concepto de movimiento está ausente, lo que concuerda con la idea de espacio sideral. 
El sub-submicroespacio semántico está integrado por los siguientes términos léxicos y sus funciones, en cada una de las cuatro frases que expresan el submacroespacio semántico:

a) (A mind poet stays in the house.) 1) Elemento determinado primario, 2) elemento determinante, 3) extensión de significado, circunstancia indicativa de espacio. 1) Consta de un término simbólico (poet), al que determina un término cuantificador (a), y acompaña un término de identificación (mind) = subjetivo. 2) Está formado por un término estático (stays), indicativo de un estado generalizado $(-0+o)$. 3) Se compone de un elemento determinado secundario (house) = término simbolico, que está relacionado con el determinante por 'in', y determinado por 'the', como término señalizador.

b) (The house is empty.) 1) Elemento determinado primario, 2) elemento determinante. 1) Consta de un término simbólico (house = espacio sideral), determinado por 'the'. 2) Se compone de un término estático (is = stays), al que, a su vez, puede considerarse como un elemento de relación entre el elemento determinado y el término determinante 'empty' = objetivo (cualidad perceptible por la vista) (the empty house).

c) ([And] it has no walls.) 1) Elemento determinado primario, 2) elemento determinante, 3) elemento determinado secundario (objeto). 'And' es un conector conjuntivo que relaciona $b$ con c. 1) Comprende un término representativo (it). 2) Consta de un término estático (has), $(-0+0)$ que lleva implícito el concepto 'propio de' (walls are improper of it). 3) Está formado por 'walls', término de contenido simbólico, al que acompaña un término determinante (no) que anula su identidad.

d) (The poem is seen from all sides, everywhere, at once.) 1) Elemento determinado primario, 2) elemento determinante, 3) extensión de significado, circunstancia indicativa de espacio. 1) Comprende un término simbólico (poem), al que determina 'the'. 2) Está constituido de un término estático (voz pasiva), $(-0+o)=$ estado genérico (is seen). 3) Comprende un elemento determinado secundario (sides) = término subjetivo relativo a un espacio, determinado por el cuantificador de carácter universal (all). 'From' une a 'sides' con el determinante, y tiene como aditamentos explicativos 'everywhere' $=$ término representativo de carácter universal e indicativo de espacio, así 
como 'at once', expresión temporal que está de acuerdo con la naturaleza universal de 'all' $y$ 'everywhere'.

El patrón rítmico tiene su expresión gráfica en: / .•• / •..•/ ..../

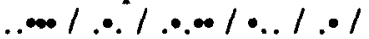

Tomando en consideración los resultados del análisis realizado, llegamos a la formulación de un esquema que sintetiza el marco creativo del poema 'As for Poets'.

- Submicrocontexto clave: apreciación genérica en la que el objeto suscita comparaciones a la manera de alegorías en la mente del poeta.

- Submacrocontexto ideológico: los componentes de nuestro entorno vital son poetas creadores de poesía: la tierra, el aire, el fuego que habita la tierra, el agua, el espacio, la mente.

- Submacroespacio semántico: situación subjetiva (en las estrofas 4, 5 y 6 se suscitan problemas geológico-bíblico, filosófico-matemático y ontológico, respectivamente).

- Submicroespacio semántico: frase alegórica en forma de circunstancia aseverativa. Los conceptos de tiempo, espacio y movimiento vienen expresados en este orden, en cuanto a la prioridad otorgada a los mismos; el tiempo está indicado por un presente configurado como norma genérica, pudiendo considerarse sin dimensión.

\section{Sub-submicroespacio semántico:}

a) Estructura: determinado primario / determinante / extensión de significado en forma de circunstancia indicativa de espacio (esta es la estructura funcional que se repite con más frecuencia).

b) Términos léxicos: (según la prioridad de frecuencia). Contenido: simbólico, subjetivo y dinámico. Funcion: determinante, de relación, modificador y representativo.

Dentro del grupo de términos determinantes están, en orden de frecuencia, 'the', los calificativos, los que determinan por identificación, los cuantificadores y la frase determinante. 
Patrón rítmico: no se observa ningún patrón distintivo en la combinación de sílabas acentuadas e inacentuadas. En el aspecto formal se produce un aumento gradual del número de versos en las estrofas 1,2 y $3(4,5,6)$, pasando a 8 versos en las tres estrofas restantes, lo que coincide con la introducción de una problemática de fondo. El número de sílabas por estrofa oscila entre 30,31 y 33, contando la estrofa 4 con un mayor número de sílabas y la 1 con uno menor; las estrofas 2 y 6 coinciden en el número de sílabas. Existe más homogeneidad en relación al número de sílabas acentuadas, oscilando entre 15, 16, 18 y 19; el menor número corresponde a la estrofa número uno (10), y la cinco y seis coinciden (15). 